\title{
An Ethnobotanical Survey for Tropical Sand Dune Support Greenbelt International Airport Yogyakarta (NYIA) Glagah Village, District Temon, Kulon Progo, Yogyakarta Indonesia
}

\section{Jati Batoro}

Biology Department, University of Brawijaya, Malang, East Java, Indonesia

\begin{abstract}
Aim of survey research on the type of Gumuk Pasir Tropik (Tropical Sand Dunes) is intended to reveal species diversity, a system of knowledge that includes the use, knowledge and fulfilment of its various needs. The survey was conducted to document plant diversity, supporting the greenbelt construction of the NYIA airport to take its documentation and sustainable development. The location of the research was conducted at NYIA Airport in Glagah village, Temon district, Kulon Progo regency, Yogyakarta Province Indonesia. The research was conducted using structural and open ended interview, direct observation and survey. Local people have a good knowledge of the diversity of plant species. However, lack of wisdom in the management resulted in natural forest sand dune ecological damage. The survey of local people in Glagah village resulted in identification of 84 plants that are presented in the paper.
\end{abstract}

Keywords: Ethnobotanical survey; Sand dune; Greenbelt; Yogyakarta

\section{Introduction}

Glagah village is limited to the south of Indonesia Sea, West of Palihan village, East times Karangwuni village and north of Kalidengen village (Figure 1). The total area of the Glagah village 603.94 hectare covers $16.64 \%$ of the total area of Temon district. The village has 8 Dukuh via Glagah, Sangkretan, Bebekan, Macanan, Kretek, Sidorejo, Bapangan and Kepek. Glagah village has a height of 1-6 MASL (Metres above sea level) with a slope level of $0-1 \%$, the air temperature is approximately $25-33^{\circ} \mathrm{C}$ and the average rainfall every year $2.342 \mathrm{~mm}$ per year.

Coastal dune environments are selective ecosystems characterized by a close interaction between abiotic and biotic factors in a dynamic balance [1]. Sand dune conservation is becoming an important plan to be implemented, on the one hand, sand dune has the potential landscape which can still developed further, and sand dune can be a barrier saviour of community behind these dunes. Sustainability and stability of sand dune can reduce tsunami attack caused by earthquake [2]. Sand dune or sand beach in Glagah village is state land which is traditionally a Pakualaman area. The area is sandy due to the combination of sand or by the wind, the sea wave's south coast (Samodra Indonesia). Studies

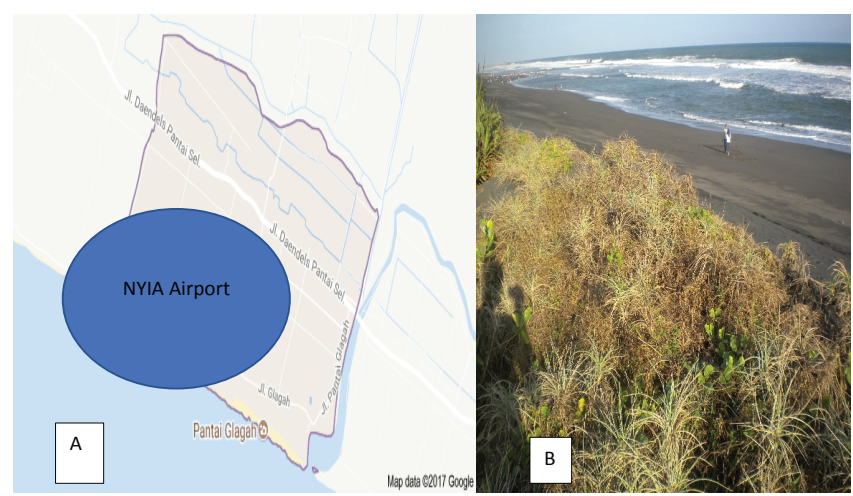

Figure 1: (A) Map of Glagah village, Temon district, Kulon Progo, Yogyakarta and NYIA airport locations of study sites; (B) Gumuk Pasir Tropik (tropical sand dune). reported the succession rate in a primary series represented a unimodal relation with total species richness. On the other hand species poor vegetation often dominated by one species, such as heathlands [3].

Local types of plants and animals are typical in the tropical sand dune area. Typical plant species include Pandanus (Pandanus tectorius), Balaran (Ipomoea pescaprae), Krandan (Canavalia maritima), Preketekan (Spinifex littorius), Widoro (Ziziphus mauritiana), Carex sp., Cimplukan (Physalis minima), Telekan (Lantana camara), Nomlang (Passiflora foetida), Alang-alang (Imperata cylindrica), Sembung (Eupatorium inulifolium), Brobos (Centrosoma pubescent), Widuri (Calotropis gigantea), Sidoguri (Sida rhombifolia), Pulutan (Triumffeta indica) [2]. Studies reported the plant family in tropical sand dunes covers e.g. Pandanaceae, Gramineae, Cyperaceae, Fabaceae, Solanaceae, Malvaceae, Rhamnaceae, Asteraceae, Apocynaceae, Euphorbiaceae, Labiatae, Pasifloraceae. Among the ten plant species in Sand dune Egypt is Tamarix aphylla, Prosopis juliflora, Acacia saligna and Atriplex nummularia show high rates of growth and significant survival rates [4].

Types cultivated of plants that have high economic value are dragon fruit (Hylocereus undatus), Lombok kriting, chili (Capsicum anuum), Semangka, watermelon (Citrullus lanatus) and Kambil, coconut (Cocos nucifera). While the types of plants are important to consider in support of green belts NYIA airport such as Pandan (Pandanus tectorius), Balaran (Ipomoea pescaprae), Krandan (Canavalia maritima), Widuri (Calotropis digantea), Carex sp., Preketekan (Spinifex littorius) and introduction species of Cemara laut (Casuarina equisetifolia), Tereside,

*Corresponding author: Jati Batoro, Faculty of Mathematics and Natural Sciences, Biology Department, University of Brawijaya, Jl. Veteran, Malang 65145, East Java, Indonesia, Tel: +62-341-575841; E-mail: jati_batoro@yahoo.co.id

Received September 06, 2017; Accepted November 24, 2017; Published December 04, 2017

Citation: Batoro J (2017) An Ethnobotanical Survey for Tropical Sand Dune Support Greenbelt International Airport Yogyakarta (NYIA) Glagah Village, District Temon, Kulon Progo, Yogyakarta Indonesia. J Coast Zone Manag 20: 452. doi: 10.4172/2473-3350.1000452

Copyright: (C) 2017 Batoro J. This is an open-access article distributed under the terms of the Creative Commons Attribution License, which permits unrestricted use, distribution, and reproduction in any medium, provided the original author and source are credited. 
Citation: Batoro J (2017) An Ethnobotanical Survey for Tropical Sand Dune Support Greenbelt International Airport Yogyakarta (NYIA) Glagah Village, District Temon, Kulon Progo, Yogyakarta Indonesia. J Coast Zone Manag 20: 452. doi: 10.4172/2473-3350.1000452

Gamal (Glyrisidia sepium), Akasia (Acacia aurifiliformis), Waru laut (Hibiscus tiliaceus) $[1,2,5]$. The industrialization with agriculture and aquaculture e.g. wetlands (tambaks) with increased use of chemical input poses create to coastal environment [6]. Amphibian diversity and reptiles at sand dune Bantul regency Yogyakarta Province identified 11 species namely Cryptoblepharus cursor, Hemidactylus frenatus, Hemidactylus garnotii, Hemidactylus platyurus and Duttaphrynus melanostictus $[7,8]$.

\section{Materials and Methods}

The study site is located at the tropical sand dune Glagah village, Yogyakarta Province and Laboratory of Plant Taxonomy Brawijaya University. The research was conducted using structural and open ended interview and direct observation or survey $[9,10]$. Based on the examination of the material survey and specimens herbarium colection Herbarium Biology Brawijaya University (H.Bio Unibraw). Fresh material colection from survey 2016-2017 in area tropical sand dune Glagah village, Temon district, Yogyakarta Indonesia. From the collection studied by the author, details and identification from dried material, except for floral and fruit sizes, which were based on dehydrated (boiled in water) material [11-14]. Terminologies and methods follows $[3,4,9,15]$. The identification and nomenclature of the listed plants were based on the Flora of Java and Flora Malesiana.

\section{Results and Discussion}

During the present research, inventarization flora local and ethnobotany follow data on 34 families, 84 plant species was collected and preserved at Herbarium Brawijaya University ( $\mathrm{H}$ Bio Unibraw). The study evidenced that agricultural plays an important role in the enhancement of the livelihoods of local peoples. Several of species and subspecies of wild plants and animal are threatened with extinction. For example: Landak (Hystrix sp.). Biological diversity conservation is and urgent coastal matter [16]. Sand dune is area an important part of coast as because the lands are first defence against the force of the ocean, protecting the diversity of native flora, fauna and the ecosystem as a whole. The other hand the understanding of tropical hydrology can serve important the realism of tropical greenbelt [17].

The types of conservation plants that need to be considered in favour of green belt are Table 1 via Pandan (Pandanus tectorius), Balaran (Ipomoea pescaprae), Krandan (Canavalia maritima), Preketek (Spinifex littorius), Akasia (Acasia aurifiliformis), Widuri (Calotropis gigantea), Gringging, gamal (Glyricidia sepium), Jambu monyet (Anacardium occidentale), Cemara laut (Casuarina equisetifolia), Jarak (Jatropha curcas), Kelor (Moringa oleifera), Teki pasir (Carex sp., Cyperus spp.), Nomlang (Passiflora foetida), Widoro (Ziziphus mauritiana), Pace (Morinda citrifolia), Cimplukan (Physalis minima) and Singkil (Premma sp.). Sharing animal species on the beach shoreline includes: Undurundur laut (Emerita spp.), jingking sapi, jingking kebo (Crustacea, Ocypodidae), Gemak (Turnix sylvaticus), Jangkrik (Gryllus campestrix), Kadal laut (Cryptoblepharus cursor), biawak (Varanus sp.), Landak (Hystrix sp.). Jingking sapi (Ocypodidae) widely used local community as a side dish (Jingking pelas). The diversity of fish species in the estuary includes Blanak (Mugil-mugil), petek (Leiognatus equulus), Bojor, Pari (Dasyatis sp.), while crustacean e.g, Shrimp, Kepiting (Brachyura sp.), Udang (Macrura sp.), Urang ayu (Cherax sp.), Siput (Molusca) and green algae.

The diversity of fauna by the local community is utilized include Undur-undur laut (Emerita sp.), fishing, duck fodder, food (peyek) while Jingking sapi (Crustaceae, Ocypodidae) is foodstuffs (pelas).
Lately tropical sand dune area of glagah village is utilized by shrimp farming. While the diversity of wild plants that are exploited are fruit or food (Physalis minima, Ziziphus mauritiana, Passiflora foetida, Canavalia maritima). Pandanus tectorius leaves used to make a hat, the root as a rope (sulur). The diversity agricultural of plants that have high economic value viz. Buah naga, dragon fruit (Hylocereus undatus), Lombok kriting, Chili (Capsicum anuum), Semangka, Watermelon (Citrullus lanatus) and Kambil or klopo, coconut (Cocos nucifera).

While the types of plants are important to consider in support of green belts NYIA airport is Pandan (Pandanus tectorius), Balaran (Ipomoea pescaprae), Krandan (Canavalia maritima), Widuri (Calotropis digantea), Carex sp., Preketekan (Spinifex littorius), Nyamplung (Calophyllum inophyllum), Keben (Baringtonia asiatica) and species introduction of Cemara laut (Casuarina equisetifolia) [18], Teresidae, gamal (Glyrisidie sepium), Akasia (Acacia auriculiformis). Tree plants species i.e., Keben (Barringtonia asiatica), Nyamplung (Callophyllum inophyllum), Pandan duri (Pandanus tectorius), Jambu monyet (Anacardium occidentale) very good for conserving green belt in tropical sand dune. Coastal sand dunes provide this opportunity, this biological diversity continuity provides an unending source of material research ecological studies $[8,18]$.

Keben (Barringtonia asiatica) family Lecythidaceae, Nyamplung (Calophyllum inophyllum) in the familia Calophyllaceae is native from East Africa, southern coastal India to Malaysia and Pandan (Pandanus tectorius) in the familia Pandanaceae, pakis haji (Cycas rumphii) familia Cycadaceae, Dudulan (Scaeveola taccada) familia Goodinaceae are tree species of flowering plants. The four species are naturally green belt on the sand beach of Alas Purwo National Park East Java. We are sure Dudulan (Scaeveola taccada), Pandanus tectorius, Baringtonia asiatica, Jambu monyet (Anacardium occidenrale) and Calophyllum inophyllum is very well grow as green belt NYIA Airport. Sand dune systems are historically mobile physically and also adaptable physiologically, and have survived many environmental changes in the past [19]. Studies reported Canavalia rosea (Sw.) D.C.; Ipomoea pes-caprae (L.) Sweet. and Schizachyrium scoparium Michx. var. lit- toralis (Nash) Hitch are almost always found growing in places with regular sand movement $[20,21]$. The function of green belt at sand dune can be a barrier savior of community, ecotorism and can reduce tsunami attack caused by earthquake. The introduction of plant species at Green belt, maybe cause aircraft flight problems with the appearance of certain bird species [22].

Information regarding their vernacular name, botanical name, family, part used and their ethno-agricultural uses are listed below starting with local name, scientific name and family name, part used and Ethno-botany uses and locations (Table 1).

\section{Conclusion}

Based on the investigation of specimen herbarium and survey were resulted that there are 34 family, 84 species are recognized. The study evidenced that agricultural plays an important role in the enhancement of the livelihoods of local peoples. A Total of 84 plants consist of tropical sand dune Glagah Village, district Temon, Kulon Progo Regency, Yogyakarta Province existing in the region. Local people have a good knowledge of the diversity of plant species. While the types of plants are important to consider in support of green belts NYIA airport is Pandan (Pandanus tectorius), Balaran (Ipomoea pescaprae), Krandan (Canavalia maritima), Widuri (Calotropis digantea), Carex sp., Preketekan (Spinifex littorius), Carex sp., Nyamplung (Calophyllum inophyllum), Keben (Baringtonia asiatica), Dudulan ((Scaeveola taccada), Jambu monyet 
Citation: Batoro J (2017) An Ethnobotanical Survey for Tropical Sand Dune Support Greenbelt International Airport Yogyakarta (NYIA) Glagah Village, District Temon, Kulon Progo, Yogyakarta Indonesia. J Coast Zone Manag 20: 452. doi: 10.4172/2473-3350.1000452

\begin{tabular}{|c|c|c|c|c|c|}
\hline S.no & Local name & Scientific name & Family & Parts used & Location and Status \\
\hline 1. & Bayam & Amaranthus hybridus L. & Amaranthaceae & Young stem, leaf & Sand dune, cultivated \\
\hline 2. & Bayam coklat & Amaranthus sp. & Amaranthaceae & Leaf, animal feed & Sand dune, wild \\
\hline 3. & Bayam duri & Amaranthus spinosus L. & Amaranthaceae & Whole, toxic & Sand dune, wild plants \\
\hline 4. & Jambu monyet & Anacardium occidentale L. & Anacardiaceae & $\begin{array}{l}\text { Stem, drinks, seed, sap fruit } \\
\text { toxic }\end{array}$ & Sand dune, cultivated, conservation* \\
\hline 5. & Widuri & Calotropis gigantea (Willd) Dryand. Ex W.T.Ait & Apocynaceae & Leaf, wrap paste & Sand dune, wild plants * \\
\hline 6. & Tapak doro & Catharathus roseus (L.) G. Don & Apocynaceae & Leaf, flower & Sand dune, cultivated \\
\hline 7. & Klopo/kambil & Cocos nucifera L. & Arecaceae & All plants, vegetable, ritual & Sand dune, cultivated \\
\hline 8. & Sembung & Eupatorium inulifolium H.B.K. & Asteracae & Leaf, flower,fertilizer & Sand dune, wild plant \\
\hline 9 & Biden & Bidens biternata (Lour) Merr. & Asteraceae & Animal feed & Sand dune, wild plants \\
\hline 10. & Tapak liman & Gynura crepidiaides L. & Asteraceae & Leaf, medecine & Sand dune, wild plants \\
\hline 11. & Telekan & Lantana camara L. & Asteraceae & Whole, toxic & Sand dune, wild \\
\hline 12. & Tempuyung & Emilia sonchifolia (L.) DC. & Asteraceae & Leaf, medecine & Sand dune, wild plants \\
\hline 13. & Wedusan & Ageratum conizoides L. & Asteraceae & Animal feed & Sand dune, wild plants \\
\hline 14. & Sundel & Borreria alata & Asteraceae & Leaf, animal feed & Sand dune, wild ${ }^{*}$ \\
\hline 15. & Klateng & Synedrella nodifora (I.) Gaertn. & Asteraceae & Leaf, animal feed & Sand dune, wild \\
\hline 16. & Nanas & Ananas camosus (L.) Merr & Bromeliaceae & Fruit & Sand dune, cultivated \\
\hline 17. & Buah naga & Hylocereus undatus (Haworth) Britton \& Rose & Cactaceae & Fruit & Sand dune, cultivated \\
\hline 18. & Nyamplung & Calophyllum inophyllum & Callophyllaceae & Stem, fruit & Sand dune, cultivated \\
\hline 19. & Pepaya & Carica papaya L. & Caricaceae & Buah, leaf, vegetable & Sand dune, cultivated \\
\hline 20. & Cemara laut & Casuarina equisetifolia L. & Casuarinaceae & Would. conservation & Sand dune, cultivated * \\
\hline 21. & Gewor & Comelina nudiflora L. & Commelinaceae & Leaf, animal feed & Sand dune, wild \\
\hline 22. & Tela rambat & Ipomoea batatas (L.) Lamk. & Convolvulaceae & Stem, leaf & Sand dune, cultivated \\
\hline 23. & Tali putri & Cuscuta sp. & Convolvulaceae & Would, conservation & Sand dune, wild* \\
\hline 24. & Semangka & Citrullus lanatus & Cucurbitaceae & Liana, fruit & Sand dune, cultivated \\
\hline 25. & Teki & Cyperus rotundus $\mathrm{L}$. & Cyperaceae & Leaf, animal feed & Sand dune, wild plant ${ }^{*}$ \\
\hline 26. & Teki & Cyperus sp. & Cyperaceae & Leaf, animal feed & Sand dune, wild plant \\
\hline 27. & Teki pasir & Cyperus melanocephalus Miq. & Cyperaceae & Leaf, animal feed & Sand dune, wild plant ${ }^{*}$ \\
\hline 28. & Buntut tikus & Acalipha indica L. & Euphorbiaceae & Leaf, medecine & Sand dune, wild \\
\hline 29. & Jarak & Jatropha curcas L. & Euphorbiaceae & Fruit, seed, toxic & Sand dune, cultivated * \\
\hline 30. & Katemas & Euphorbia heterophylla & Euphorbiaceae & Leaf, toxic, animal feed & Sand dune, wild plants \\
\hline 31. & Patikan & Euphorbia hirta & Euphorbiaceae & Leaf, toxic, animal feed & Sand dune, wild \\
\hline 32. & Telo kaspo & Monihot utilisima Pohl. & Euphorbiaceae & Radix, stem, leave, food & Sand dune, cultivated \\
\hline 33. & Meniran & Phyllanthus niruri L. & Euphorbiaceae & Leaf, fruit, medicine & Sand dune, wild ${ }^{*}$ \\
\hline 34. & Akasia & Acasia auriculiformis A. Cunn.ex Bth & Fabaceae & Would, leaf, conservation & Sand dune, cultivated, consernation * \\
\hline 35. & Benguk & Mucuna pruriens (L.) DC. & Fabaceae & Seed, toxic, tempe & Sand dune, cultivated \\
\hline 36. & Brobos & Centrosoma pubescent Bth. & Fabaceae & Leaf, animal seed & Sand dune,wild \\
\hline 37. & Gringging, gamal & Glyricidia sepium (Jacq.) Kunth ex Walp & Fabaceae & Stem, leave, animal feed & Sand dune, cultivated * \\
\hline 38. & Kacang brol & Arachis hypogaea L. & Fabaceae & Leaf, seed & Sand dune, culivated \\
\hline 39. & Kacang tolo & Phasolus vulgaris $\mathrm{L}$. & Fabaceae & Fruit, vegetable & Sand dune, cultivated \\
\hline 40. & Krandan & Canavalia marritima (Aubl.) Urb. & Fabaceae & Seed: tempe, flower: pecel & Sand dune, wild* \\
\hline 41. & Mlandingan & Leucaena glauca Bth. & Fabaceae & Fruit, seed, latex medecine & Sand dune, cultivated \\
\hline 42. & Orok-orok besar & Crotalaria mucronata Desv & Fabaceae & Daun, animal feed & Sand dune, wild plants \\
\hline 43. & Orok-orok kecil & Crotalaria striaca DC & Fabaceae & Daun, animal feed & Sand dune, wild plants \\
\hline 44. & Rendetan & Desmodium sp. & Fabaceae & Leaf, animal feed & Sand dune, wild \\
\hline 45. & Riwilkop & Mimosa pudica L. & Fabaceae & Toxic & Sand dune, wild \\
\hline 46. & Tom & Indigofera sumatrana Gaertn & Fabaceae & Leaf, color & Sand dune, wild* \\
\hline 47. & Alang-alang & Imperata cylindrica (L.) Beauv & Gramineae & Leaf, medecine, animal feed & Sand dune, wild \\
\hline 48. & Wulu asu & Fimbristylis sp. & Gramineae & Leaf, animal feed & Sand dune, wild \\
\hline 49. & Rumput gajah & Pennisetum purpureum L. & Gramineae & Leaf, animal feed & Sand dune, cultivated \\
\hline 50. & Empritan & Eragrostis amabilis O.K & Gramineae & Leaf, animal feed & Sand dune, wild plants \\
\hline 51. & Grinting & Cynodon dactylon (L.) Pers. & Gramineae & Leaf, animal feed & Sand dune, wild \\
\hline 52. & Jagung & Zea mays L. & Gramineae & Fruit, leaf, food, animal feed & Sand dune, cultivated \\
\hline 53. & Kawatan & Eleusin indica Gaertn & Gramineae & Leaf, animal feed & Sand dune, wild plants \\
\hline 54. & $\begin{array}{l}\text { Preketekan, } \\
\text { rumput angin }\end{array}$ & Spinifex littorius Merr. & Gramineae & Flower, toys & Sand dune, wild * \\
\hline 55. & Sereh & Adropogon citratus DC & Gramineae & Whole plant, flavoring & Sand dune, cultivated \\
\hline 56. & Rumput & Fimbristylis cymosa & Gramineae & Leaf, animal feed & Sand dune, wild \\
\hline 57. & Kemangi & Ocimum basilicum L. & Labiatae & Leaf, young stem, vegetable & Sand dune, cultivated \\
\hline 58. & Sengketan & Hyptis suaveolens (.) Poit & Labiatae & Flower & Sand dune, wild \\
\hline
\end{tabular}


Citation: Batoro J (2017) An Ethnobotanical Survey for Tropical Sand Dune Support Greenbelt International Airport Yogyakarta (NYIA) Glagah Village, District Temon, Kulon Progo, Yogyakarta Indonesia. J Coast Zone Manag 20: 452. doi: 10.4172/2473-3350.1000452

\begin{tabular}{|c|c|c|c|c|c|}
\hline 59. & Leng-lengan & Leucas aspera (Willd) L. & Lamiaceae & Herb, leaf, medecine & Sand dune, Wild \\
\hline 60. & Singkil & Premma sp. & Lamiaceae & Habitus, Conservation & Sand dune, wild \\
\hline 61. & Pulutan & Urena lobata L. & Malvaceae & Toxic, fruit & Sand dune, wild plants \\
\hline 62. & Sidaguri & Sida rhombifolia L. & Malvaceae & Leaf, animal feed & Sand dune, wild plants * \\
\hline 63. & Tewel, nongko & Artocarpus heterophyllus L. & Moraceae & $\begin{array}{l}\text { Stem, fruit, vegetable, animal } \\
\text { feed }\end{array}$ & Sand dune, cultivated \\
\hline 64. & Kelor & Moringa oleifera & Moringiaceae & Young eaf & Sand dune, cultivated \\
\hline 65. & Gedang, & Musa paradisiaca L. cv Ambon & Musaceae & Fruit, stem, latex, flower, leaf & Sand dune, cultivated \\
\hline 66. & Jambu klutuk & Psidium quajava L. & Myrtaceae & Fruit, leaf, medecine & Sand dune, cultivated \\
\hline 67. & Pandan duri & Pandanus tectorius Soland. Ex Park. & Pandanaceae & Leaf, roof, mats, conservation & Sand dune, wild plants * \\
\hline 68. & Nomlang & Passiflora foetida L. & Passifloraceae & Fruit, toxic & Sand dune, wild plants * \\
\hline 69. & Katu & Sauropus androgynus & Phyllantaceae & Leaf, fruit & Sand dune, wild \\
\hline 70. & Lang-layangan & Drynaria calomelanos & Polypodiaceae & Steril leaf kites & Sand dune, wild plants \\
\hline 71. & Widoro & Ziziphus mauritiana Lamk. & Rhamnaceae & Fruit & Sand dune, wild plants* \\
\hline 72. & Pace & Morinda citrifolia L. & Rubiaceae & Fruit, medecine & Sand dune, wild ${ }^{*}$ \\
\hline 73. & Balaran & Ipomoea pescaprae (L.) R.Br & Solanaceae & Stem, roof, conservation & Sand dune, wild plants * \\
\hline 74. & Ipo. & Ipomoea sp. & Solanaceae & Herb & Sand dune, wild \\
\hline 75. & Cimplukan & Physalis minima $\mathrm{L}$. & Solanaceae & Fruit, medecine & Sand dune, wild plants* \\
\hline 76. & Lombok kriting & Capsicum anuum L. & Solanaceae & Fruit, chili & Sand dune, cultivated \\
\hline 77. & Lombok rawit & Capsicum frutescents $\mathrm{L}$. & Solanaceae & Fruit, chili, vegetable & Sand dune, cultivated \\
\hline 78. & Ranti & Solanum torvum SW & Solanaceae & $\begin{array}{c}\text { Fruit, leave, vegetable, } \\
\text { medecine }\end{array}$ & Sand dune, wild \\
\hline 79. & Terong & Solanum melongena L. & Solanaceae & Fruit, vegetable & Sand dune, cultivated \\
\hline 80. & Tomat & Lycopersicon esculentum L. & Solanaceae & Fruit, vegetable & Sand dune, cultivated \\
\hline 81. & Pulutan & Triumffeta indica Auct. Non Lamk. & Tiliaceae & Consevation & Sand dune, wild \\
\hline 82. & Pecut kuda & Stachypeta jamaicensis (L.) Vahl. & Verbenaceae & Leaf, medecine & Sand dune, wild plants \\
\hline 83. & Lipi & Phyla nodiflora (L.) Righ & Verbenaceae & Fruit, conservation & Sand dune, wild plants * \\
\hline 84. & Laos & Alpinia galanga (L.) Wild & Zingiberaceae & $\begin{array}{l}\text { Rhizoma, medecine, } \\
\text { vegetable }\end{array}$ & Sand dune, cultivated \\
\hline
\end{tabular}

Table 1: The different plants used in the five common are tabulated.

(Anacardium occidentale), waru (Hibiscus tiliaceus) and introduction of Cemara laut, Cemara udang (Casuarina equisetifolia), Teresidae, Gamal (Glyrisidie sepium), Akasia (Acacia aurifiliformis).

\section{Acknowledgements}

The authors are grateful to the sponsoring the project and thanks to Faculty of Mathematics and Natural Sciences, University of Brawijaya for supporting this research. The researcher also gives thanks to the local communities of local people Glagah village, Kulon Progo. Last but not least, we also thank Trilaksono for the support from the leader Glagah village.

\section{References}

1. Fenu G, Cogoni D, Ferrara S, Pinna MS, Bacchetta G, et al. (2011) Relationships between coastal sand dune properties and plant community distribution: The case of Is Arenas (Sardinia). Taylor \& Francis Group, UK. pp: 586-602.

2. Budiyanto (2011) Landscape conservation technology. J Land Indo 3: 2-14

3. Isermann M (2011) Patterns in Species diversity during succession of coastal dunes. J Coast Res 27: 661-671.

4. Backer CA, Bakhuizen VDB (1968) Flora of java. N.V. wolter noordhoff. Batavia, Jakarta.

5. Batoro J, Rahardi B (2017) The basics of plant systematics. UB Press Publishers, Malang. pp: 60-88.

6. Clark JR (1996) Coastal zone management.

7. Cotton CM (1996) Ethnobotany: Principle and applications. John Wiley \& Sons, Chichester, New York. pp: 4-12.

8. Crowford RMM (2009) The biology of coastal sand dunes. Ann Bot 104: 6-8.

9. Davis PH, VH Heywood (1963) Principles of angiosperm taxonomy. Oliver \& Boyd, London. pp: 556
10. Hoffman B, Gallaher T (2007) Importance indices in ethnobotany. Ethnobotany Research \& Applications 5: 201-208.

11. Keng K (1989) Malayan seed plants.

12. Lee D, Oreopoulos L, Huffman GJ, Rossow WB, (2013) The precipitation characteristics of ISCCP tropical weather states. Journal AMS 4: 715-718.

13. Martinez, Moreno (1996) Growth and survival in six tropical sand dune species from the gulf of Mexico. J Coast Res 12: 406-419.

14. Maun MA (2009) The biology of coastal sand dunes. Ann Bot 104: 6-7.

15. Misak R, Draz MY (1997) Sand drift control of selected coastal and desert dunes in Egypt: Case studies. J Arid Env 35: 17-28.

16. Nugroho AW, Sumardi (2009) Amelioration tread for shrimp firm establishment (Casuarina equisetifolia L.) on gumuk pasir pantai (site amelioration for establishment of Casuarina equisetifolia L. at Coastal Sand Dune).

17. Poyyamoli G, Padmavathy K, Balachandran N (2011) Coastal sand dunes, vegetation structure, Diversity and disturbance in Nallavadu village, Puducherry India. Asian J Water Environ Pollut 8: 115-122.

18. Rambo AT (1983) Conceptual approaches to human ecology. East-West Environment and Policy Institute, USA. Research Report 14: 6-18.

19. Vanstenis CGGJ (1988) Flora for school in Indonesia. Pradnya Paramita Jakarta.

20. Westphal E, Jansen PCM (1989) Plant resource of South-East Asia Pudoc Wageningen.

21. Balgooy MMJ (1987) Manual of herbarium taxonomy theory and practice.

22. Qurniawan TF, Eprilurahman R (2013) Diversity of amphibians and reptiles of sandstone in yogyakarta special province. Zoo Indonesia 22: 9-16. 